\title{
Fenologia de uma espécie arbórea em ecótono Caatinga / Cerrado no sul do Piauí
}

\author{
Phenology of tree species in Caatinga / Cerrado boundary in southern Piaui \\ Rejane Tavares Botrel ${ }^{1}$, Danúbia Rejane Silva Brito ${ }^{2}$, Waldeídes Castro Sousa $^{3}$, Anderson Marcos Souza $^{4}$, Alan Cauêe
Holanda $^{5}$
}

Resumo: O objetivo deste trabalho foi caracterizar a fenologia de uma população natural de Terminalia fagifolia Mart, localizada em ecótono entre os Biomas Cerrado e Caatinga, no município de Bom Jesus, sul do Estado do Piauí. Vinte indivíduos, com distância mínima de 20 metros entre si, foram marcados, utilizando GPS. Os dados fenológicos foram coletados durante um ano e as fenofases observadas foram brotação, desfolhamento, floração e frutificação. Para análise dos dados foram utilizados índices de atividade, intensidade e sincronia, além da correlação de Spearman que avaliou dados fenológicos em conjunto com variáveis ambientais. A análise fenológica indicou presença de todas as fenofases durante o período de estudos, sendo o desfolhamento observado continuamente, variando apenas em seu grau de intensidade. A população estudada apresentou sincronia perfeita entre os indivíduos nas fenofases desfolhamento e brotação. Quanto à correlação de Spearman, os resultados não indicaram correlações significativas entre fenofases e variáveis ambientais.

Palavras-chaves: Fenofases. Correlação de Spearman. Índice de sincronia.

Abstract: The aim this work was to characterize the phenology of Terminalia fagifolia Mart. in a Cerrado/Caatinga boundary , in Bom Jesus, Piauí, Brazil. Twenty individuals were marked using GPS. The phenological study was conducted for 1 year and were observed the phenophases sprouting, leaf loss, flowering and fruiting. For analysis of phenological data were used the index of activity, the synchronism index and correlation of Spearman. The phenological analysis indicated the presence of all phenophases during the study period, with defoliation observed continuously, varying only in their degree of intensity. The study population had a perfect synchrony between individuals to defoliation and sprouting. Regarding the Spearman correlation the results did not indicate significant correlations between phenophases and environmental variables.

Key words: Phenophases. Spearman correlation. Synchronism index.

\footnotetext{
*Autor para correspondência

Recebido para publicação em 15/05/2015; aprovado em 20/06/2015

${ }^{1}$ Doutora em Ciências Vegetais, Departamento de Ciências Vegetais, Universidade Federal Rural do Semiárido, rtbotrel@ufersa.edu.br

${ }^{2}$ Mestre em Ciências Florestais, Núcleo de educação à distância, Universidade Estadual do Piauí, danubiarejane@ hotmail.com

${ }^{3}$ Graduada em Engenharia Florestal, Setor Meio Ambiente da Itaueira Agropecuária S/A, waldeidescastro@itaueira.com.br

${ }^{4}$ Doutor em Engenharia Florestal, Departamento de Engenharia Florestal, Universidade de Brasília, andermsouza@yahoo.com.br

${ }^{5}$ Doutor em Ciências Florestais, Departamento de Ciências Vegetais, Universidade Federal Rural do Semiárido, Alan.holanda@ufersa.edu.br
} 


\section{INTRODUÇÃO}

O sul do Estado do Piauí é considerado uma das últimas fronteiras agrícolas dentro do bioma Cerrado. Com o fortalecimento da agricultura nesta região, grandes áreas foram desmatadas, o que levou a concentração das populações vegetais naturais remanescentes em maciços florestais isolados.

$\mathrm{O}$ estado tem vivenciado uma ocupação acelerada do Cerrado, iniciada na década 70 com a implantação de grandes projetos agropecuários (pecuária e cajucultura). Já na década de 90, esse processo foi intensificado por meio da implementação de grandes projetos para a produção de grãos, principalmente soja, voltada para a exportação (AGUIAR e MONTEIRO, 2005).

Uma particularidade a respeito da vegetação da região sul do estado do Piauí é a presença distinta de áreas de Caatinga e Cerrado, bem como ecótonos desses dois biomas, considerados áreas de contato, pois não existe aí uma vegetação característica local, e sim uma associação de dois ou mais tipos ecológicos diferentes (CEPRO, 1996).

As áreas de transição entre Caatinga e Cerrado têm sido pouco investigadas e, por sua situação de ecótono, com grande diversidade de espécies, merecem atenção especial. Além disso, o conhecimento das características ecológicas das espécies arbóreas destas áreas de transição é relativamente importante, devido a possibilidade de utilização destas espécies, adaptadas a duas formações vegetais distintas, em projetos de recuperação e recomposição de áreas degradadas.

A fenologia vegetal, que estuda a periodicidade e a duração de eventos biológicos como a foliação, a floração e a frutificação, fornece informações que permitem a análise da dependência de tais eventos em relação a fatores bióticos e abióticos (RAMOS e SANTOS, 2006). Assim, dados provenientes de estudos fenológicos servem de subsídio para outros estudos, tal como aqueles referentes à produção e tecnologia de sementes, pois permitem a indicação da melhor época para colheita e plantio das espécies (SANTOS e TAKAKI, 2005).

Terminalia fagifolia Mart., espécie da família Combretaceae, distribui-se geograficamente no Brasil nas regiões Nordeste (BA, CE, MA, PI), Centro-Oeste (DF, GO, MS, MT) $\quad$ e

(MG)

(floradobrasil.jbrj.gov.br/2010/FB006913).

Relativamente abundante e amplamente distribuída nos ecotónos entre Caatinga e Cerrado no sul do estado do Piauí, T. fagifolia apresenta boa empregabilidade na carpintaria e como lenha e carvão, além de potencial paisagístico e medicinal (LORENZI, 2002). Além disso, a espécie citada pode ser uma alternativa a ser utilizada na recuperação de áreas degradadas. A espécie, que também recebe os nomes populares cachaporra-do-gentio e capitão-do-seco (LORENZI, 2002), é localmente conhecida no município de Bom Jesus-PI, como catinga-de-porco e, a despeito de sua plasticidade de utilização, foi alvo, até o momento, de poucos estudos.

Portanto, o objetivo deste estudo foi caracterizar o comportamento fenológico de uma população de Terminalia fagifolia Mart. em um trecho de vegetação em ecótono entre os biomas Caatinga e Cerrado no sul do Estado do Piauí, bem como verificar se existe correlação entre fenofases e variáveis ambientais.

\section{MATERIAL E MÉTODOS}

Os dados para esta pesquisa foram coletados entre março de 2009 e março de 2010 , em uma população natural de T. fagifolia , localizada no município de Bom Jesus, sul do Estado do Piauí, na propriedade rural conhecida como Fazenda Chapada das Éguas, cujas coordenadas são 9०02'23”S e 4423'33'W. Com altitude de 360m, a propriedade é caracterizada por uma vegetação de transição entre os biomas Caatinga e Cerrado.

Dados meteorológicos da região indicam temperaturas mínimas de $18{ }^{\circ} \mathrm{C}$, máximas de $36{ }^{\circ} \mathrm{C}$ e clima quente e semiúmido (BANDEIRA et al., 2010). A precipitação pluviométrica média anual é definida dentro do Regime Equatorial Continental e o período chuvoso estende-se de novembro a dezembro e de abril a maio. $\mathrm{O}$ trimestre mais úmido corresponde aos meses de dezembro, janeiro e fevereiro (BANDEIRA et al., 2010).

Para a caracterização fenológica, 20 indivíduos adultos de T. fagifolia, com distância mínima de 20 metros entre si, foram escolhidos e visitados quinzenalmente, seguindo o método proposto por Morellato e Leitão-Filho (1990). Todos os indivíduos foram identificados com plaquetas de alumínio e localizados geograficamente com a utilização do Sistema de Posicionamento Global (GPS).

Durante as visitas a campo foram observadas fenofases vegetativas (desfolhamento e brotação) e reprodutivas (floração e frutificação). Todas as fenofases foram analisadas utilizando métodos qualitativo (Índice de atividade) e quantitativo (Índice de intensidade). O Índice de atividade indica a presença ou ausência do evento fenológico. Já o Índice de intensidade estima a intensidade de tal evento (FOUNIER, 1974). De acordo com o Índice de intensidade de Fournier (1974), as fenofases são caracterizadas pela sua maior ou menor frequência em um determinado período de tempo e para isso é adotada a seguinte escala: 0 (zero)ausência do fenômeno; 1 - ocorrência de 1 a 25\%; 2 ocorrência de 26 a 50\%; 3- ocorrência de 51 a 75\%; 4ocorrência de 76 a $100 \%$. Mensalmente, fez-se a soma dos valores de intensidade obtidos para todos os indivíduos e dividiu-se pelo valor máximo possível (número de indivíduos multiplicado por quatro). O valor obtido, multiplicado por 100, corresponde a uma proporção (BENCKE e MORELLATO, 2002).

Cabe ressaltar que por floração entendeu-se a presença de flores, não havendo distinção entre botão floral e flor aberta, e a frutificação foi determinada no período em que os frutos encontravam-se verdes, maduros ou secos.

Para análise dos dados fenológicos foi também utilizado o Índice de sincronia. Morellato et al. (1990) afirmam que a sincronia entre os indivíduos de uma população pode ser estimada pelo método no qual é constatada a presença ou ausência da fenofase, ou seja, a atividade daquele evento na população. Quanto maior o número de indivíduos manifestando a fenofase ao mesmo tempo, maior será a sincronia desta população (BENCKE e MORELLATO, 2002).

Segundo Ruiz e Alencar (1999), a sincronia é a ocorrência simultânea de um evento particular. O índice de sincronia foi calculado de acordo com Augspurger (1983) (1), tendo inicialmente o resultado referente à sincronia de um determinado indivíduo com seus co-específicos ou o índice de 
sincronia individual (xi), e como resultado posterior o índice de sincronia da população (Z) (2).

$$
X_{i}=\left(\frac{1}{n-1}\right) x\left(\frac{1}{f_{i}}\right) x \sum_{j=1}^{n} e_{j \neq 1}
$$

Onde: ej= número de meses que os indivíduos i e j estão na mesma fenofase; fi= número de meses em que o indivíduo i está na fenofase; $n=$ número de indivíduos na população.

$$
Z=\frac{1}{n} \sum_{i=1}^{n} x_{i}
$$

Onde: $\mathrm{n}=$ número de indivíduos na população; $\mathrm{xi}=$ sincronia do indivíduo i com seus co-específicos.

Quando $X=1$ ocorre sincronia perfeita, ou seja, todos os meses do evento fenológico observado no indivíduo i, coincidem com todos os meses desse mesmo evento nos outros indivíduos da população $\mathrm{j} \neq \mathrm{i}$. Quando $\mathrm{X}=0$ não ocorre sincronia, indicando que não existe sobreposição entre qualquer mês de evento no individuo i com qualquer outro indivíduo na população (RUIZ e ALENCAR, 1999).

Para investigar se a variação das fenofases ao longo do período estudado acompanha a variação dos componentes climáticos (temperatura, umidade e precipitação) foi calculada a correlação de Spearman por meio do programa Statistical Analysis System (SAS, 2003). Para isso foram utilizados os dados mensais referentes às variáveis ambientais (temperatura, umidade e precipitação), registradas durante o período de coleta de dados fenológicos (março/2009 a março/2010) disponibilizados pela Universidade Federal do Piauí, Campus Professora Cinobelina Elvas. Cabe ressaltar que no referido período o regime hídrico foi atípico, com baixa precipitação $(845,0 \mathrm{~mm})$ e má distribuição durante o ano.

\section{RESULTADOS E DISCUSSÃO}

A análise dos dados fenológicos coletados indicou que para o desfolhamento de $T$. fagifolia os picos de atividade e de intensidade não obtiveram respostas semelhantes. Considerando o Índice de atividade, a perda da parte aérea foi concentrada entre março/2009 a setembro/2009, voltando a aumentar em novembro/2009 e entre janeiro/2010 a março/2010 (Figura 1).

De acordo com Bencke e Morellato (2002), o pico de atividade pode estar relacionado a características endógenas e a fatores abióticos que podem influenciar a fisiologia vegetal, determinando ou restringindo o período de ocorrência das fenofases. No entanto, ao analisar aqui os eventos fenológicos em conjunto com variáveis ambientais por meio da correlação de Spearman, nenhum resultado significativo foi registrado, indicando localmente uma independência entra as variáveis analisadas (Tabela 1). Desta forma, podemos reforçar que a população de $T$. fagifolia investigada neste trabalho, parece apresentar um padrão de desfolhamento regulado por questões endógenas.
Figura 1 - Representação fenológica do desfolhamento de Terminalia fagifolia Mart., no período de março/2009 a março/2010, em uma população natural, em área de transição Cerrado -Caatinga no município de Bom Jesus, Piauí.

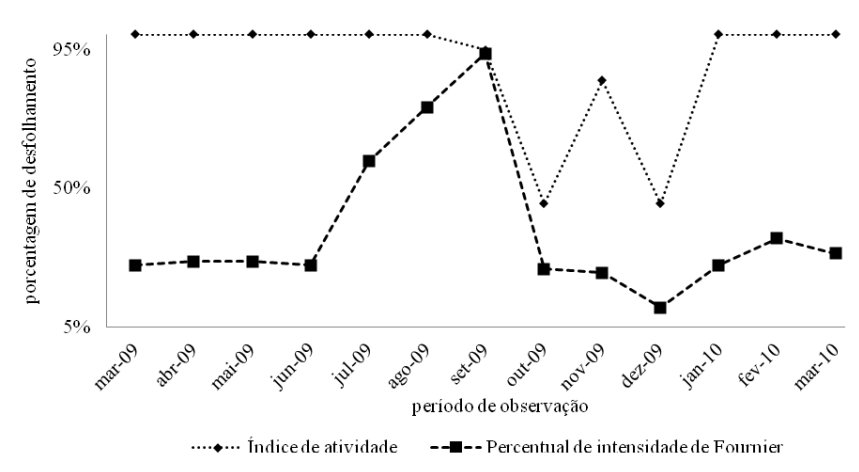

É importante destacar que o desfolhamento de $T$. fagifolia teve como característica marcante (observações pessoais) a perda do limbo de forma gradual, iniciando com pequenos orifícios semelhantes à deteriorações causadas por lagartas e evoluindo até o desaparecimento completo da folha. Este fato explica os longos períodos de perda de folhas registrados durante a pesquisa. Portanto, o desfolhamento foi a fenofase mais presente nas observações de campo, totalizando doze meses de evento, ou seja, todo o ano de observação, sendo diferenciado apenas pela intensidade do evento.

Tabela 1: Coeficiente de correlação de Spearman (r) entre fenofases e variáveis ambientais para uma população natural de Terminalia fagifolia Mart., em área de transição Cerrado Caatinga no município de Bom Jesus, Piauí.

\begin{tabular}{cccc}
\hline \multirow{2}{*}{ Eventos } & \multicolumn{3}{c}{ Variáveis } \\
\cline { 2 - 4 } & Temperatura & Umidade & Precipitação \\
\hline Desfolhamento & $-0,04868$ & $-0,11988$ & $-0,20932$ \\
Brotação & 0,47049 & $-0,33809$ & 0,08402 \\
Frutificação & $-0,15876$ & $-0,34189$ & $-0,53205$ \\
Floração & 0,49785 & $-0,43581$ & $-0,04830$ \\
\hline
\end{tabular}

Analisando o Índice de intensidade nota-se um aumento do desfolhamento entre os meses de julho/2009 a setembro/2009, com pico de intensidade em setembro/2009 (Figura 1). De acordo com Bencke e Morellato (2002), a falta de coincidência entre os picos de atividade e intensidade tende a ser mais evidente nas fenofases vegetativas, devido ao incremento cumulativo na magnitude da fenofase (em \% de indivíduos ou de intensidade) ser lento e gradual.

A perda de folhas, em ambientes com restrição hídrica como a Caatinga, é uma estratégia fisiológica das plantas para reduzir a quantidade de água eliminada pela transpiração. No entanto, a resposta dos indivíduos analisados (figura 1) não condiz totalmente com essa premissa sugerindo que, apesar do período mais intenso de perda foliar ter coincidido com o período mais seco da região, o ecótone entre Caatinga e Cerrado parece imprimir particularidades à população de $T$. fagifolia aqui estudada.

Em relação à análise da sincronia de eventos fenológicos na população de $T$. fagifolia analisada, é importante frisar que, mesmo apresentando um desfolhamento atípico, o fato dos indivíduos permanecerem desfolhados por mais de um mês, caracteriza a espécie como caducifólia (NEVES et al., 
2010). Isso indica que a espécie apresentou uma alta sincronia para este evento, o que é comprovado pelos valores do índice de sincronia entre os indivíduos e para a população de $T$. fagifolia encontrados (Tabela 2). Dos indivíduos observados, $45 \%$ apresentaram sincronia perfeita $(1,0)$ para o desfolhamento e os demais obtiveram alta sincronia $(0,8 ; 0,9)$. Consequentemente, a alta sincronia entre os indivíduos resultou em uma ótima sincronia para a população com valor bem próximo ao que indica uma sincronia perfeita $(0,9)$.

Tabela 2 - Índice de sincronia dos eventos fenológicos entre indivíduos (Xi) e dentro da população natural (X) de Terminalia fagifolia Mart., em área de transição Cerrado Caatinga no município de Bom Jesus, Piauí.

\begin{tabular}{|c|c|c|c|c|c|c|c|c|}
\hline \multirow{2}{*}{$\begin{array}{r}\text { In } \\
\text { d. }\end{array}$} & \multicolumn{2}{|c|}{ Desfolhamento } & \multicolumn{2}{|c|}{ Brotação } & \multicolumn{2}{|c|}{ Floração } & \multicolumn{2}{|c|}{ Frutificação } \\
\hline & $\mathrm{Xi}$ & $\mathrm{X}$ & $\mathrm{Xi}$ & $X$ & $\mathrm{Xi}$ & $\mathrm{X}$ & $\mathrm{Xi}$ & $\mathrm{X}$ \\
\hline 1 & 0,9 & & 0,9 & & 0,0 & & 0,4 & \\
\hline 2 & 0,9 & & 0,9 & & 0,0 & & 0,0 & \\
\hline 3 & 1,0 & & 0,7 & & 0,1 & & 0,3 & \\
\hline 4 & 1,0 & & 0,6 & & 0,1 & & 0,2 & \\
\hline 5 & 0,8 & & 0,8 & & 0,0 & & 0,2 & \\
\hline 6 & 0,9 & & 0,6 & & 0,0 & & 0,4 & \\
\hline 7 & 0,9 & & 0,9 & & 0,0 & & 0,3 & \\
\hline 8 & 0,9 & & 0,5 & & 0,0 & & 0,0 & \\
\hline 9 & 1,0 & & 0,6 & & 0,0 & & 0,3 & \\
\hline 10 & 1,0 & 0,9 & 0,5 & 0,7 & 0,1 & 0,02 & 0,4 & 0,2 \\
\hline 11 & 1,0 & & 0,8 & & 0,0 & & 0,3 & \\
\hline 12 & 0,9 & & 0,8 & & 0,0 & & 0,0 & \\
\hline 13 & 0,9 & & 0,6 & & 0,0 & & 0,4 & \\
\hline 14 & 0,9 & & 0,6 & & 0,0 & & 0,4 & \\
\hline 15 & 0,9 & & 0,5 & & 0,0 & & 0,0 & \\
\hline 16 & 0,9 & & 0,4 & & 0,0 & & 0,0 & \\
\hline 17 & 1,0 & & 0,7 & & 0,0 & & 0,0 & \\
\hline 18 & 1,0 & & 0,6 & & 0,0 & & 0,0 & \\
\hline 19 & 1,0 & & 0,6 & & 0,0 & & 0,0 & \\
\hline 20 & 1,0 & & 0,7 & & 0,0 & & 0,0 & \\
\hline
\end{tabular}

Em campo foi ainda observado que as árvores mais baixas apresentaram menor porcentagem de desfolhamento. Isso poderia sugerir que, como estas se encontram relativamente mais sombreadas pelas árvores mais altas, a menor incidência direta do sol, torna a referida fenofase menos gradual, quando comparadas com os indivíduos mais altos. No entanto, esta hipótese carece de estudos mais aprofundados para se confirmar ou não.

A fenofase brotação, que ocorreu entre julho/2009 e fevereiro/2010, apresentou respostas semelhantes para o pico de atividade e de intensidade, tendo como período representativo os meses de outubro/2009 e dezembro/2009 (Figura 2).
Figura 2 - Representação fenológica da brotação de Terminalia fagifolia Mart., no período de março/2009 a março/2010, em uma população natural, em área de transição Cerrado -Caatinga no município de Bom Jesus, Piauí.

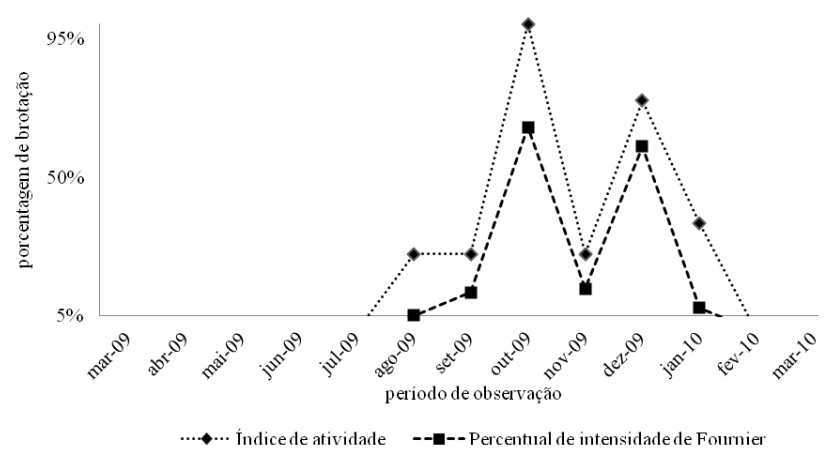

É válido salientar que se entendeu por brotação o período inicial de surgimento das folhas até o momento em que toda a copa apresentava os brotos.

Em alguns trabalhos pode-se perceber a clara relação da fenofase brotação com o local de ocorrência dos indivíduos estudados e, até mesmo, com outras fenofases. Fagundes et al. (2007), estudando a brotação de uma espécie típica do Cerrado (Caryocar brasiliense Cambess), cita que a produção de folhas novas variou entre os habitats estudados e os meses de avaliação. Quando o autor analisa a interação entre habitats e os meses de estudo, também percebeu variação na fenofase brotação. Já Marques e Oliveira (2004) observando fenofases em Florestas de Restinga perceberam que a renovação de folhas em sua área de estudo foi um evento sazonal, com queda concentrada entre os meses de outubro e dezembro e brotação imediatamente após, entre novembro e janeiro, ambas no início do período superúmido.

Certamente que as espécies possuem características inerentes à sua conformação genética. No entanto, considerando que o fenótipo de tais espécies pode refletir a adaptação das plantas em diferentes habitats, o padrão de brotação encontrado para T. fagifolia no ecótono estudado, pode representar sua estratégia fisiológica. Analisando em conjunto, brotação e desfolhamento, nota-se a perda do limbo foliar de forma gradual até que as copas das árvores percam todas as folhas senescentes e o início da brotação sobrepondo o final do referido evento. A fenofase brotação se inicia em julho/2009 que, usualmente, não corresponde ao início do período chuvoso no sul do Piauí. Assim, mesmo que não ocorra deciduidade típica na espécie estudada, pode-se inferir que a redução de área foliar diminui a perda de água que parece ser suficiente para o início da brotação. Cabe salientar que o pico de intensidade (Figura 2) da emissão de folhas novas ocorreu no início do período chuvoso, indicando que à medida que o período seco se torna menos crítico, a brotação atinge seu ápice.

Para a fenofase brotação verificou-se a inexistência de sincronia perfeita entre os indivíduos observados e seus coespecíficos (tabela 2), sendo que a espécie apresentou a maior parte de indivíduos com valor de sincronia entre médio e alto $(0,6 ; 0,9)$. Essa variação no valor de sincronia entre os indivíduos resultou em um valor médio $(0,7)$ de sincronia para a população (Tabela 1 ).

A ótima sincronia para a população, no que diz respeito ao desfolhamento, sugere eficiência da estratégia fisiológica 
para evitar perda de água. No entanto, em relação a sincronia da fenofase brotação, nota-se que os indivíduos não manifestaram o evento de forma sincrônica. Isso leva a crer que não somente o conteúdo de água disponível no solo, mas o conteúdo de água existente na própria planta poderia estar regulando a brotação.

A fenofase floração, ao contrário do desfolhamento e brotação, ocorreu por um período curto entre setembro/2009 e outubro/2009 (Figura 3) e durante as observações nota-se que não houve muita diferença na quantidade de flores de um mês para o outro. Este resultado é importante, pois indica um período relativamente curto para polinização dos indivíduos da população estudada. Isto poderia comprometer a perpetuação da espécie $T$. fagifolia no ambiente estudado, a despeito da quantidade regular de flores por indivíduo durante os dois meses de ocorrência do evento.

Figura 3 - Representação fenológica da floração de Terminalia fagifolia Mart., no período de março/2009 a março/2010, em uma população natural, em área de transição Cerrado -Caatinga no município de Bom Jesus, Piauí.

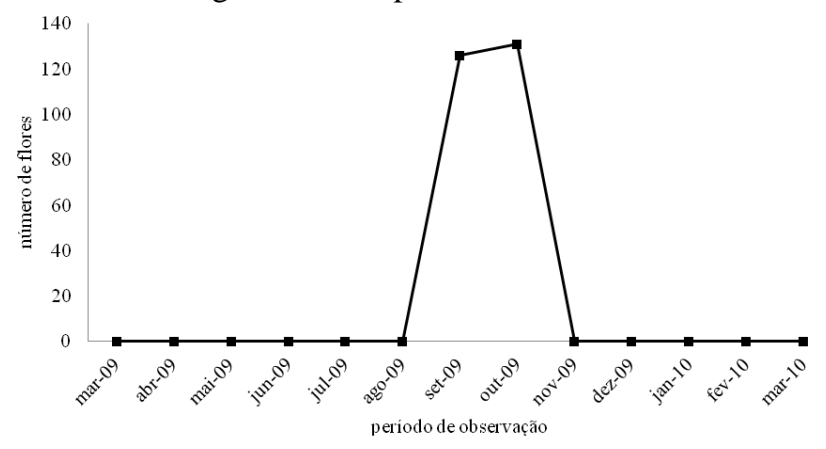

No que se refere ao pico de atividade, os resultados deste trabalho para a fenofase floração coincidem com aqueles apresentados por Almeida et al. (1998) para a espécie T. fagifolia em áreas de Cerrado. No entanto, o autor cita que o evento se manifesta de julho a janeiro, sugerindo um resultado atípico para o presente trabalho ou uma particularidade da espécie em ecótonos entre Caatinga e Cerrado. Lorenzi (2002), considerando a distribuição geográfica da espécie, também apresenta dados semelhantes que fortalecem os resultados aqui apresentados. Por outro lado, Figueiredo (2008) estudando espécies arbóreas em área de Cerrado, registrou resultados para $T$. fagifolia bem diferentes dos encontrados nesta pesquisa, com período de floração entre janeiro e maio.

No que diz respeito à sincronia, a floração foi a fenofase que apresentou menores índices entre os indivíduos, sendo registrados valores baixos $(0,1)$ e em apenas três deles. Assim, a falta de sobreposição do evento entre os indivíduos resultou em baixa sincronia também para a população $(0,02)$ (tabela 2).

Segundo Ruiz e Alencar (1999) índices de sincronia para a fenofase floração são importantes para caracterização do processo reprodutivo da espécie estudada. Desta forma, a polinização acaba sendo afetada por essa falta de sincronia.

A fenofase frutificação se manifestou em um intervalo de tempo significativo, entre março/2009 e outubro/2009 (Figura 4). Os frutos de T. fagifolia são secos, indeiscentes e alados, o que sugere a dispersão anemocórica (ALMEIDA et al., 1998). Assim a disponibilidade de frutos por um longo período é importante para a eficiência da dispersão dos frutos. Comparando os resultados aqui obtidos com os de Almeida et al. (1998), verifica-se semelhança entre os períodos de ocorrência do evento. Figueiredo (2008) também apresenta resultados coincidentes com os da presente pesquisa, concentrando a frutificação entre março e junho. A mensuração feita para o material reprodutivo teve como pico de intensidade o mês de agosto/2009 (Figura 4), onde foi possível encontrar uma maior quantidade de frutos nos indivíduos observados.

Figura 4 - Representação fenológica da frutificação de Terminalia fagifolia Mart., no período de março/2009 a março/2010, em uma população natural, em área de transição Cerrado -Caatinga no município de Bom Jesus, Piauí.

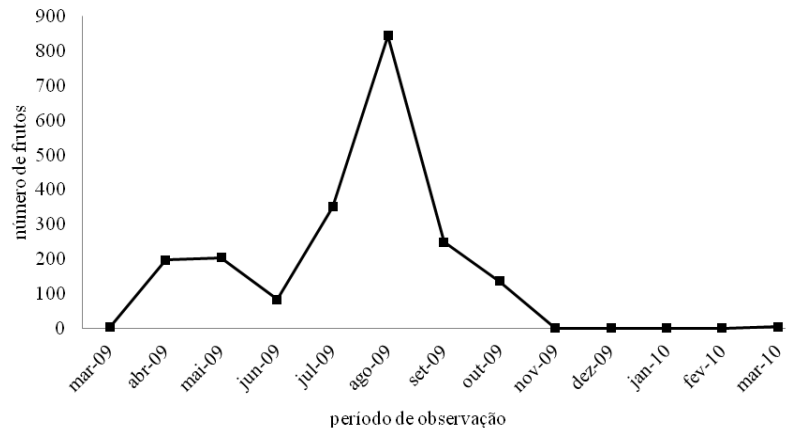

A sincronia entre os indivíduos e a população de $T$. fagifolia para o evento reprodutivo frutificação foi baixa, ou seja, não houve sobreposição entre o mês de frutificação de alguns indivíduos com seus co-específicos. A falta de sobreposição gerou valores considerados de médio a baixo $(0,4 ; 0,2)$ para os indivíduos e baixo para a população $(0,2)$ (Tabela 1). Como as plantas apresentam diferentes estratégias fenológicas para garantir a atração de dispersores de sementes, o sucesso da dispersão das sementes de uma espécie pode ser resultado de uma frutificação em massa por um curto período ou poucos frutos maduros por dia durante um período longo. Assim, a falta de sincronia encontrada para os indivíduos de $T$. fagifolia pode ser positiva se considerarmos a ocorrência de frutos em diferentes períodos de tempo.

\section{CONCLUSÕES}

A espécie Terminalia fagifolia Mart. apresentou calendário fenológico bem definido quanto às suas fenofases. A fenofase desfolhamento se destacou por ter sido observada durante todo o período de estudo, variando apenas em seu grau de intensidade. A população apresentou uma sincronia perfeita entre os indivíduos para o desfolhamento e para a brotação. Os índices de correlação de Spearman indicaram correlações não significativas entre fenofases e fatores abióticos, o que sugere uma possível estabilidade fenológica na população estudada, independente dos fatores abióticos analisados. No entanto, devido ao regime hídrico atípico no ano de estudo, com baixa precipitação $(845,0 \mathrm{~mm})$ e má distribuição durante o ano, não se pode afirmar que as fenofases seriam ou não influenciadas pelas varáveis ambientais em anos com diferentes regimes hídricos. 


\section{REFERÊNCIAS BIBLIOGRÁFICAS}

AGUIAR, T. DE J. A. DE; MONTEIRO, M. DO S. L. Modelo agrícola e desenvolvimento sustentável: a ocupação do Cerrado piauiense. Ambiente e Sociedade, v. 8 , n. 2 , jul/dez, 2005 .

ALMEIDA, S. P.; PROENÇA, C.E.B.; SANO, S.M.; RIBEIRO, J.F. Cerrado: espécies vegetais úteis. Planaltina, DF: Embrapa-CPAC, 1998. 464 p.

AUGSPURGER, C. K. Phenology, flowering, synchrony and fruit set of six neotropical shrubs. Biotropica, Zurich, v. 15, n. 4, p. 257-267, dez. 1983.

BANDEIRA, E. G.; ALVES, C. M. D.; MELO, L. F. S. Análise temporal por imagens landsat da expansão agrícola no município de Bom Jesus -PI. In: III Simpósio brasileiro de ciências geodésicas e tecnologias de geoinformação, 2010, Recife. Anais do III Simpósio brasileiro de ciências geodésicas e tecnologias de geoinformação Recife, Universidade Federal de Pernambuco, Recife, 2010.

BENCKE, C. S. C.; MORELLATO, L. P. C., Comparação de dois métodos de avaliação da fenologia de plantas, sua interpretação e representação. Revista Brasileira de Botânica, São Paulo, v.25, n.3, p.269-275, set, 2002.

CEPRO. Piauí: Caracterização do quadro natural. Teresina, PI: Fundação CEPRO, 1996. 116p.

FAGUNDES, M.; ARAÚJO, L. S.; NUNES, Y. R. F. Efeitos do Estágio Sucessional do Habitat na Fenologia do Pequizeiro (Caryocar brasiliense: Caryocaraceae). Revista Brasileira de Biociências, Porto Alegre, v. 5, supl. 1, p. 144-146, jul. 2007

FIGUEIREDO, P. S. Fenologia e estratégias reprodutivas das espécies arbóreas em uma área marginal de Cerrado, na transição para o semi-árido no nordeste do Maranhão, Brasil. Revista Trópica - Ciencias Agrárias e Biológicas, Chapadinha, v.2, n.2, p.8-22, 2008.

FOURNIER, L. A. Un método cuantitativo para la medición de características fenológicas em árboles. Turrialba, San Jose, v.24, n.4, 1974.

LORENZI, H. Árvores brasileiras: Manual de identificação e cultivo de plantas arbóreas nativas do Brasil. Nova Odessa, SP: Instituto Plantarum, 2002. v.2, 97p.

MARCHIORETTO, M. S.; MAUHS, J.; BUDKE, J. C. Fenologia de espécies arbóreas zoocóricas em uma floresta psamófila no sul do Brasil. Acta Botanica Brasílica, São Paulo v.21, n.1, p. 193-201, 2007.
MARQUES, M. C. M.; OLIVEIRA, P. E. A. M. Fenologia de espécies do dossel e do sub-bosque de duas Florestas de Restinga na Ilha do Mel, sul do Brasil. Revista Brasileira de Botanica, São Paulo, v. 27, n.4, p.713723, out.-dez, 2004.

MORELLATO, L.P.C; LEITÃO FILHO, H.F., RODRIGUES, R.R.; JOLY, C.A.. Estratégias fenológicas de espécies arbóreas em floresta de altitude na Serra do Japi, Jundiaí, São Paulo. Revista Brasileira de Biologia, São Paulo, n.50, p.149-162, 1990.

MORELLATO, L.P.C.; LEITÃO-FILHO, H.F. Estratégias fenológicas de espécies arbóreas em floresta mesófila na serra do Japi, Jundiaí, São Paulo. Revista Brasileira Biologia, São Paulo, n. 50, p. 463-173, 1990.

NEVES, E.L. das; FUNCH, L.S.; VIANA, B.F. Comportamento fenológico de três espécies de Jatropha (Euphorbiaceae) da Caatinga, semi-árido do Brasil. Revista Brasileira de Botânica, São Paulo, v.33, n.1, p.155-166, jan./mar, 2010.

PEREIRA, M. R. N.; TONINI, H. Fenologia da andiroba (Carapa guianensis Aubl., Meliaceae) no Sul do Estado de Roraima. Ciências florestal, Santa Maria, v.22, n.1, p.47-58, jan./mar. 2012.

RAMOS, F. N.; SANTOS, F. A. M. Floral visitors and pollination of Psychotria tenuinervis (rubiaceae): distance from the anthropogenic and natural edges of an atlantic forest fragment. Biotropica, São Paulo, n. 38, p.383-389, 2006.

RUIZ, J. E. A.; ALENCAR, J. da C.,Interpretação fenológica de cinco espécies de Chrysobalanaceae na reserva florestal Adolpho Ducke, Manaus, Amazonas, Brasil. Acta Amazônica, Manaus, v.29, n.2, p.223-242, 1999.

SANTOS, D. L. dos; TAKAKI, M. Fenologia de Cedrela fissilis Vell. (Meliaceae) na região rural de Itirapina, SP, Brasil. Acta Botanica Brasílica, São Paulo, 19(3):625632. 2005.

SANTOS, F. P.; FERREIRA, W. M. Estudo fenológico de Davilla elliptica St. Hill. e Qualea grandiflora Mart. em uma área de Cerrado sentido restrito em Porto Nacional, Tocantins. Interface, Porto Nacional, v. 01, p. 03-14, 2012.

SAS Institute Inc., SAS/STAT. User's guide: statistic, version 9.1.2. Cary, NC: SAS Institute INC, 2004. 